\title{
Chronic illness and mental strain: The protective role of partners with time since illness onset
}

\author{
Jack Lam \\ The University of Queensland, Australian Research Council Centre of Excellence for \\ Children and Families Over the Life Course and Institute for Social Science Research, \\ Australia \\ Francisco Perales \\ The University of Queensland, Australian Research Council Centre of Excellence for \\ Children and Families Over the Life Course and Institute for Social Science Research, \\ Australia
}

(Received December $2017 \quad$ Revised March 2018)

http://dx.doi.org/10.14301/Ilcs.v9i3.483

\section{Abstract}

Chronic conditions are associated with large personal, familial and social costs, and have deleterious effects on individuals' mental health. Drawing on the stress process model, we theorise and test how the presence of a partner moderates the extent to which living with a chronic condition affects mental health, and whether any protective effects change with time since illness onset, or differ between men and women. Our empirical analyses rely on nationally representative, panel data for Australia ( $\mathrm{n}-180,000$ observations) and panel regression models. Being in a partnership, particularly in a marriage, is associated with better mental health amongst all individuals, but more so amongst the chronically ill. This advantage remains beyond the year of illness onset, and is of a comparable magnitude for men and women. These findings bear important implications for mental health in modern societies experiencing rapid population ageing, a rising prevalence of chronic illness, and declining marriage rates.

\section{Keywords}

Chronic illness; ageing; Australia

\section{Introduction}

Chronic conditions are associated with large monetary and social costs to individuals and their families, and constitute a sizeable burden to the health care system (AlHW, 2014; Essue, Kelly, Roberts, Leeder, \& Jan, 2011). In addition, such conditions are becoming more prevalent, largely as a consequence of an ageing population. As people live longer, they have both increasing chances of ever being diagnosed with a chronic illness and of experiencing such illness over a prolonged period of time. ${ }^{1}$ Compared to acute illnesses, chronic conditions are slow in their progression and often result in the emergence of new or increasingly pronounced functional limitations to which individuals must adjust (Dunlop, Lyons, Manheim, Song, \& Chang, 2004). This is important, as undertaking large, recurrent and long-lasting life adjustments is a mentally straining process, and can have detrimental impacts on individuals' mental wellbeing (Holahan, Holahan, \& Belk, 1984; Serido, Almeida, \& Wethington, 2004). Consistent with this, a breadth of international research has documented robust links between living with a chronic illness and poor mental health (Hollingshaus \& Utz, 2013; Polsky et al., 2005; Pudrovska 2010).

Within this context, it is important that we further our understanding of the factors that buffer the negative mental health consequences of living 
with a chronic illness. Previous research has made some inroads in this regard, providing evidence that social support and networks are important moderators of the relationship between chronic conditions and mental wellbeing (Schafer \& Koltai, 2015). Partners are important sources of social support, and have the potential to buffer the impact of stressors associated with living with a chronic condition (Berkman \& Glass, 2000; Cohen, 2004). In this paper, we extend previous studies of the protective effects of partnerships on health by incorporating a temporal component (Liu \& Umberson, 2008; Rendall et al., 2011). Specifically, we consider whether and how any protective effects of having a partner on mental health evolve with time since illness onset. Because there are well-established gender differences in spousal caregiving, whereby wives typically provide more care than husbands (Noël-Miller, 2010), we also pay attention to potential gender differences.

Drawing on the stress process model, we develop a set of hypotheses and test these using nationally representative, panel data from the Household Income and Labour Dynamics in Australia (HILDA) Survey and random-effect panel regression models. Our findings indicate that being in a partnership, and particularly being in a marriage, is associated with comparatively better mental health amongst the chronically ill. This advantage remains beyond the year of illness onset, and is of a comparable magnitude for men and women. These findings bear important implications for mental health trends in modern societies in which the population is ageing, chronic illness is prevalent, and the incidence of marriage is in decline.

\section{Theoretical background}

\section{The stress process and the dynamics of chronic illness and mental strain}

Stress process theory and an associated body of evidence link individuals' social relations to their health outcomes (Pearlin, Menaghan, Lieberman, \& Mullan, 1981; Pearlin, 1989). The stress process model pays particular attention to how 'stressors' (i.e. sources of stress) lead on to stress manifestations, of which poor mental health is a key one (Pearlin et al., 1981). Life events can be stressors, particularly if they are undesirable, uncontrollable or unscheduled, and if they lead on to life strains. The onset and maintenance of a chronic illness is a recognised stressor: the onset of a chronic condition is disruptive and uncontrollable, and it often leads to functional limitations on the self that produce prolonged strains. Consistent with this, a robust body of empirical evidence documents that individuals living with a chronic condition commonly report elevated levels of depressive symptoms (Hollingshaus \& Utz, 2013; Polsky et al., 2005; Pudrovska 2010) and anxiety disorders (Clarke \& Currie, 2009). Given this, we hypothesise that:

Hypothesis 1: Living with a chronic condition will be associated with poor mental health.

While this is not a new proposition, we revisit it in our panel data to (i) provide novel evidence that applies to the contemporary Australian context, and (ii) 'set the scene' to develop more innovative hypotheses that consider moderation by marital status and longitudinal dependencies. Concerning the latter, the stress process model also pays attention to different types of stressors depending on their temporal nature, distinguishing between 'eventful experiences' (i.e. the occurrence of discrete events that are disruptive to the self) and 'chronic strains' (i.e. the presence of relatively continuous problems) (Avison \& Turner, 1988). The experience of chronic illness combines elements of both: while illness onset qualifies as an 'eventful experience', the physical pain and functional limitations associated with the condition qualify as 'chronic strains'. This suggests different scenarios concerning the association between chronic conditions and mental health and its possible changes with time since illness onset (De Ridder, Geenen, Kuijer, \& van Middendorp, 2008; Kristofferzon, Lofmark, \& Carlsson, 2003; Polsky et al., 2005). On the one hand, it is possible to expect a 'cumulative disadvantage' scenario: beyond the initial diagnosis, living with a chronic condition could become more stressful (De Ridder et al., 2008). The functional limitations associated with chronic conditions often change as the illness progresses, requiring not just initial but continuous adjustments in multiple life domains (Stanton, Revenson \& Tennen, 2007). This is particularly so for degenerative chronic conditions, which entail more strenuous and costly adjustments over time (Cho, et al. 1998). As time since illness onset progresses, psychological distress amongst the chronically ill may also emerge due to protective resources being eroded, lost or depleted. For example, the costs of medication could produce 
financial constraints that in turn affect mental wellbeing. Altogether, this line of reasoning suggests that:

Hypothesis 2a: Chronic illnesses will have a less negative effect on mental health in the year of their onset than in subsequent years.

On the other hand, it is possible that the emotional consequences associated with the initial disruption produced by illness onset can take a particularly large emotional toll (Newman, Steed, \& Mulligan, 2004). Learning that one has a chronic condition is a form of 'biographical disruption' affecting individuals' sense of self and personal identity (Bury, 1982), as they take on a new social role as a 'sick person' (Mechanic \& Volkart, 1961) and become exposed to disease discourses that affect their emotions (Perry, 2011). For example, cancer diagnoses are often followed by external inquiries about what people had done to become sick, or whether they would adequately manage their illness (Willig, 2011). In this early stage after diagnosis, individuals may not yet have gathered the resources necessary to buffer the functional limitations associated with the health condition, or developed a capacity to mentally process and cope with the event. Pearlin's stress process model describes this phase as "a period of readjustment during which the system struggles to re-establish a homeostasis" and highlights how "the struggle for readjustment can be wearing and exhausting, and under these conditions the organism becomes outstandingly vulnerable to stress and its physical and psychological consequences" (Pearlin et al., 1981: 339). As time since illness onset progresses and resources that help buffer the negative consequences of the illness are obtained, the psychological wellbeing of chronically ill individuals could improve. Consistent with this, studies such as Polsky et al., (2005) find that depressive symptoms decrease with time since illness onset. Given this, and in contrast with Hypothesis 2a, we also hypothesise that:

Hypothesis 2b: Chronic illnesses will have a more negative effect on mental health in the year of their onset than in subsequent years.

\section{The protective role of partners and time since illness onset}

The stress process model highlights the importance of resources that can be invoked by individuals to protect themselves from the detrimental effects of stressors (Pearlin et al., 1981;
Pearlin, 1989). Such resources include coping mechanisms (mastery and self-esteem) and social support. Partners are amongst the most integral relationships in adults' lives, and constitute a key source of social support and personal wellbeing (Coombs, 1991; House, Landis, \& Umberson, 1988). Partnerships are particularly important as sources of social support against stressors because they are characterised by extensive relations and frequent interaction, and high levels of involvement and concern (Pearlin et al., 1981). Consistent with these theoretical propositions, an extensive body of evidence demonstrates the mental health benefits of partnerships and marriages, and the factors upon which this relationship is dependent (e.g. relationship duration and marital quality) (Frech \& Williams, 2007; Williams, 2003). The positive health effects of having a partner often remain after accounting for the selection of healthy individuals into relationships (Guner, Kulikova \& Llull, 2014; Murray, 2000; Rendall et al., 2011). In addition to its direct effects on mental health, being in an intimate relationship can also modify the severity of the detrimental consequences that negative life events and transitions can have on individuals' mental wellbeing. That is, partners can act as 'buffers'. For example, the negative mental health impacts of unemployment and job loss are felt less strongly by partnered individuals (Kasl \& Jones, 2000). Generally, these protective effects are attributed to the emotional and financial support provided by one's partner, as well as their provision of healthrelated social control (August \& Sorkin, 2010; Rendall et al., 2011; Umberson, 1992) and social integration and attachment (Umberson, 1987).

In the case of chronic illness and mental health, partners are an especially important source of support that may help alleviate the negative mental health effects of living with a chronic condition. On the one hand, partners can provide intangible types of support, such as emotional availability, being present when important information about the health condition is passed on, backing up important decisions (e.g. about treatment or employment participation), assist in managing medication use, and/or gathering information about how to best address functional limitations associated with the condition. On the other hand, partners can also provide more tangible types of support to meet the needs associated with the illness. This includes financial support to cover medication costs, help 
confronting reductions in labour income due to the illness, and assistance in daily activities (e.g. help walking, carrying things, or performing routine household tasks and housework). Individuals with chronic conditions receiving either kind of support may feel that they have the resources necessary to make successful life adjustments following the onset of a chronic illness, and suffer less psychological strain as a result (Berkman \& Glass, 2000; Cohen \& Wills, 1985; Helgeson \& Cohen, 1996; Stanton, Revenson, \& Tennen, 2007).

Some authors also report an additional buffer provided by marriage relative to de facto relationships. This is attributed to lower relationship commitment, satisfaction and expectations amongst individuals in de facto relationships compared to their married counterparts (Wiik, Bernhardt, \& Noack, 2009; Wiik, Keizer, \& Lappegård, 2012), which may result in a comparative advantage amongst married individuals in eliciting care and support from their partners (Noël-Miller, 2011). Based on these theoretical propositions, we hypothesise that:

Hypothesis 3: The negative mental health impacts of chronic conditions will be stronger amongst unpartnered than partnered individuals - particularly married individuals.

The role of a partner as a support source for people who live with a chronic condition may however change with time since illness onset (NoëlMiller, 2010). First, there is evidence that marital quality decreases with declines in spousal health (Booth \& Johnson, 1994), and such changes may have implications for the willingness and ability of spouses to provide care and assistance. Second, partners who act as caregivers over a prolonged period of time may experience stress and burnout as a result (Adelman, Lyubov, Tmanova, Dion, \& Lachs, 2014; Pitceathly and Maguire, 2003), which would also make them less likely to continue providing care, the same amount of care, or care of the same quality. Third, unpartnered individuals may adjust to living with a chronic condition through self-care, as well as drawing on friends and family for support, rendering the presence of a partner less salient as time since illness onset elapses. We thus develop a final research hypothesis:

Hypothesis 4: The protective effect of being partnered on the mental health of individuals living with a chronic condition will decline with time since illness onset.

\section{Accounting for gender differences}

The stress-buffering effects associated with having a partner may operate through emotional support (e.g. listening, showing affection, dealing with a partner's frustration) or instrumental support (e.g. help moving, cleaning oneself, preparing meals). However, there are important gender differences in the propensity to provide these supports, with women being more likely than men to do so (Moen, Robison \& Fields, 1994). This is the product of the persistence of traditional gender ideologies and gender divisions of paid and unpaid labour (England, 2010), as well as of the normative association of 'emotion work' (i.e. intentional activities performed to improve another person's emotional wellbeing) with femininity (Thomeer, Reczek, \& Umberson, 2015). Consistent with this, empirical research has demonstrated that women tend to receive less support from their partners when they are ill than men (Kristofferzon, Lofmark, \& Carlsson, 2003; Noël-Miller, 2010). Furthermore, female partners are typically the primary source of support for male partners who are ill, but when female partners are ill they are more likely to receive outside help; for example, from adult children or formal care services (Katz, Kabeto, \& Langa, 2000; Spitze \& Ward, 2000). Women who live with chronic illnesses are also less likely than men to nominate their partner as their primary caregiver (Allen, Goldscheider, \& Ciambrone, 1999). It is therefore possible that chronically ill women are less likely to receive support from their partners than chronically ill men, and we may thus expect smaller differences in mental health by marital status amongst women with chronic illness. This is consistent with findings indicating that, in elderly couples, marriage protects men but not women from depressive symptoms (Bierman, 2012).

Given the gender differences established in the literature, we will examine whether the expected associations outlined in our research hypotheses differ across men and women. In the next section, we introduce the data used to undertake our empirical analyses.

\section{Dataset and variables}

To examine the longitudinal associations between chronic conditions, marital status and mental health we leverage panel data from the 
Household, Income and Labour Dynamics in Australia (HILDA) Survey. The HILDA Survey is a longitudinal survey comprising the period 20012016, and its sample is largely representative of the Australian population in 2001. In Wave 1 (2001), 7,682 households were selected for participation in the study following a complex, multi-stage sampling strategy. Information was then collected from all individuals age 15 and over living in the selected households using a mixture of self-complete questionnaires and computer-assisted face-to-face interviews. Since then, these individuals have been followed over time and data have been collected from them on an annual basis. New participants can join the HILDA Survey after the initial wave if they live in participating households and turn 15 years of age, or if they begin living with an existing sample member. If the new panel member marries or has a child with an existing sample member, he/she would also be tracked over time. The HILDA Survey has sample sizes ranging from 12,408 to 17,612 across its waves, and remarkably low attrition rates for international standards. For example, the attrition rate in its latest wave (Wave 15) was circa 5\% (Summerfield et al., 2016). For more detailed information on the HILDA Survey, see Watson and Wooden (2012) and Summerfield et al. (2016).

The HILDA Survey is a unique dataset to test our research hypotheses because: (i) it contains repeated measurements of the mental health, chronic conditions, and marital status of the same individuals over time, which enables us to examine their longitudinal associations; (ii) it tracks individuals annually and over a prolonged observation window (15 years), which permits us to observe change in marital status and time since illness onset; (iii) it contains information from a large sample of individuals, which provides adequate statistical power; and (iv) it is nationally representative at baseline, which makes our results likely generalisable to the Australian population.

We use information from individuals who participated in waves 1 to 15 of the HILDA Survey, irrespective of whether or not these individuals reported having a chronic condition upon entering the panel. Our analytic sample includes all personyear observations without missing data on model variables. Where there was missing data in an independent or dependent variable, we dropped the person-year record containing the missing data, but retained other person-year records from the same respondent. Of 217,917 person-year observations in the HILDA Survey, 24,436 (or 11\%) have missing data on model variables. Of these, 24,251 (or 99\%) lack information on mental health, as this is asked within a self-complete questionnaire that incurs higher non-response than the face-toface instrument. A bias analysis was undertaken by comparing the records dropped due to missing data and those retained for analysis. Given the very large sample size, differences in the sample means of the records dropped and retained were generally statistically significant at $p<0.05$ in $t$-tests ( 20 of 22 comparisons). However, differences in means were generally small in magnitude. Mean differences amounting to $10 \%$ or more of the variable's standard deviation indicated that, in the analytic sample, there was overrepresentation of individuals who were older, married, highly educated, nonIndigenous Australian or born in an Englishspeaking country and had two or more children, and underrepresentation of individuals who were younger, single, poorly educated, Indigenous Australian or born in a non-English-speaking country, and childless. In addition, derivation of a longitudinal measure of chronic conditions entails dropping information from individuals' first observation ( $n=12,917$ observations; details below). Our final sample comprises 180,564 observations (96,138 for women and 84,426 for men) from 26,734 individuals $(13,859$ women and 12,877 men).

Our outcome of interests is respondents' mental health. To operationalise this, we use the Mental Health Inventory (MHI-5), which is a subscale of the Medical Outcomes Questionnaire Short-Form Health Survey (SF-36) (Ware \& Sherbourne, 1992). The MHI-5 is a validated measure that is routinely used in the survey literature to capture overall mental health levels. It is an additive scale constructed by combining responses to five questions, which collectively tap the four major mental health dimensions - anxiety, depression, loss of emotional/behavioural control, and psychological wellbeing (Ware \& Sherbourne, 1992). Questions ask how often in the past four weeks the respondent had (i) "been a nervous person", (ii) "felt so down in the dumps that nothing could cheer them up", (iii) "felt calm and peaceful", (iv) "felt down" and (v) "been a happy person". Possible responses are: (i) "all of the time", (ii) "most of the time", (ii) "a good bit of the time", (iv) 
"some of the time", (v) "a little of the time" and (vi) "none of the time". As is typical in the literature, the resulting additive scale is then transformed so that it ranges from 0 (worst possible outcome) to 100 (best possible outcome). In the HILDA Survey, the MHI-5 questions are collected via a self-complete questionnaire, as to ensure that respondents' answers are not biased by the presence of an interviewer (e.g. through social desirability bias). In our sample, the average score in the MHI-5 is 74.2 $(S D=17.2), 73$ amongst women $(S D=17.5)$ and 75.4 amongst men (SD=16.7).

The HILDA Survey includes information, updated annually, on whether respondents have a chronic condition. Specifically, as part of the face-to-face interview, HILDA Survey participants are asked the following yes/no question: "Do you have any longterm health condition, impairment or disability (such as these) that restricts you in your everyday activities, and has lasted or is likely to last for 6 months or more, and cannot be corrected by medication or medical aids?" Respondents are then presented with a showcard containing a list of 15 conditions, which are used as prompts. However, the question wording does not limit responses to these conditions. We first peruse this information to create a 'base' variable capturing the concurrent presence of a chronic condition. This is a dichotomous variable that takes the value zero if the respondent does not have a chronic condition (73\%; $n=131,277)$, and the value one if the respondent has a chronic condition (27\%; $n=49,287$ observations). We then develop a more complex and insightful longitudinal measure of chronic conditions that splits time around illness onset into three stages. This takes the value zero if the respondent does not have a chronic condition (73\%; $n=131,277$ ), the value one if the respondent has a chronic condition that emerged since the previous year $(9 \% ; n=16,998)$, and the value two if the respondent has a chronic condition that emerged more than one year before $(18 \% ; n=32,289)$. Individuals who previously reported having a chronic condition but no longer do so score zero in this variable. It is not possible to determine whether the chronic conditions observed in Wave 1 had their onset within the previous year, and so all observations from this survey wave are excluded from analysis.

The HILDA Survey contains time-varying information on individuals' marital status across all of its waves. We use this information to create a discrete variable separating each respondent observation into: (i) married $(50 \%, n=89,477)$, (ii) in a de facto relationship $(13 \%, n=24,675)$, (iii) divorced, separated or widowed $(14 \%, n=24,635)$, and (iv) single (never married) $(23 \%, n=41,777)$. This variable is used as a potential moderator of the relationship between the presence of a chronic condition and mental health in our regression models.

In our multivariate regression models we control for a set of factors known to be correlated with both the presence of a chronic condition and mental health, and which have been used in previous studies (Hollingshaus \& Utz, 2013; Schnittker, 2005). These include respondent's age (in years, uncentered), number of children (none, one, two or more), highest educational qualification (below school Year 12, school Year 12, professional qualification, degree or higher), employment status (employed, unemployed, not in the labour force), household financial-year, disposable, regular income (adjusted to 2015 prices and expressed in AU\$10,000s), and respondent's ethno-migrant background (Australian, not Indigenous; Australian, Indigenous; migrant, main English-speaking country; migrant, other country). Gender-specific means and standard deviations for all model variables are shown in table 1. 
Table 1. Gender-specific sample means/percentages and standard deviations

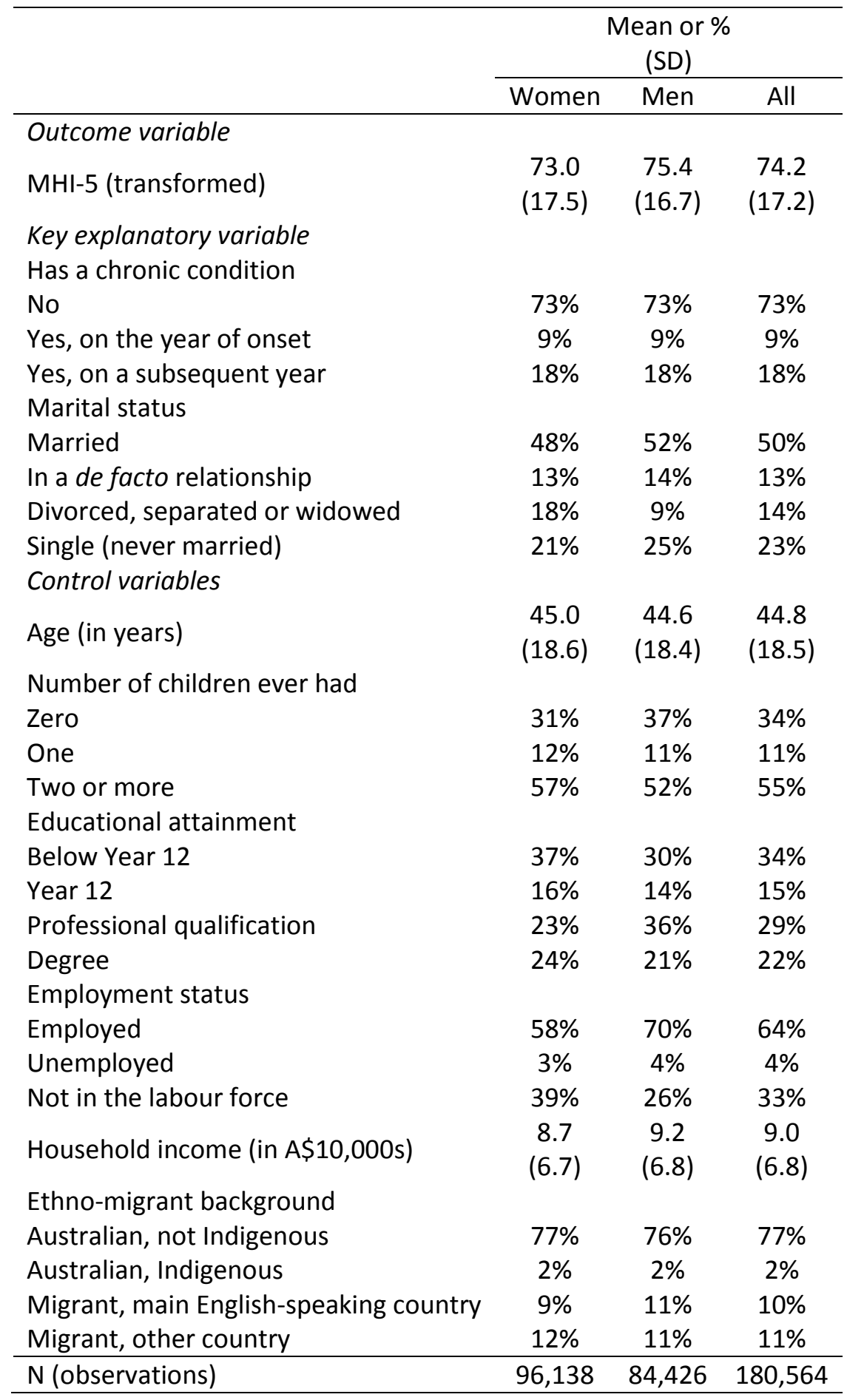

Notes: HILDA Survey data, 2002-2015. Statistics are for person-year observations across all survey waves. 


\section{Estimation method and analytic approach}

To examine the multivariate associations between chronic conditions, marital status and mental health, we estimate random-effect panel regression models that leverage the panel structure of the HILDA Survey data. ${ }^{2}$ These random-effect models account for the nesting of observations within the same individuals, and their coefficients are estimated using a weighted average of the within-individual and between-individual variability in the panel data (Wooldridge 2010). Our base model, model 1 , can be expressed as:

$\mathrm{H}_{\mathrm{it}}=\mathrm{C}_{\mathrm{it}} \beta_{1}+\mathrm{M}_{\mathrm{it}} \beta_{2}+\mathrm{X}_{\mathrm{it}} \beta_{3}+\mathrm{Z}_{\mathrm{i}} \beta_{4}+\mathrm{u}_{\mathrm{i}}+\mathrm{e}_{\mathrm{it}}$

where subscripts $i$ and $t$ denote individuals and time periods, respectively; $H$ is a continuous variable capturing mental health; $C$ is a categorical variable capturing the experience of a chronic condition that enters the model as a set of dummy variables; $M$ is a categorical marital status variable that also enters the model as a set of dummy variables; $X$ and $Z$ are vectors of time-varying and time-constant explanatory variables, respectively; and the $B s$ are the model coefficients to be estimated. There are two distinct error terms in this model: $u$ is a personspecific error (i.e. a random intercept or random effect) capturing individual-specific heterogeneity, and is assumed to come from a normal distribution with a mean of zero and to be orthogonal to the observable variables, while $e$ is the usual stochastic error term in regression. ${ }^{3}$ The coefficients on the chronic condition and marital status dummy variables in this model are used to test hypotheses 1 and 2. The coefficients on the two dummy variables capturing time since illness onset and the three dummy variables denoting marital status that appear in the models are compared through Wald tests.

To test hypotheses 3 and 4 we estimate an additional model, model 2 . This includes interaction terms between the measures of marital status and chronic conditions:

$\mathrm{H}_{\mathrm{it}}=\mathrm{C}_{\mathrm{it}} \beta_{1}+\mathrm{M}_{\mathrm{it}} \beta_{2}+\left(\mathrm{C}_{\mathrm{it}} * \mathrm{M}_{\mathrm{it}}\right) \beta_{3}+\mathrm{X}_{\mathrm{it}} \beta_{4}+\mathrm{Z}_{\mathrm{i}} \beta_{5}+\mathrm{u}_{\mathrm{i}}+\mathrm{e}_{\mathrm{it}}$

The estimated coefficients on the interactions between the chronic condition $(C)$ and marital status $(M)$ variables, i.e. $B_{3}$ in equation (2), are used to assess whether there is evidence of moderation (hypothesis 3). To test whether moderation differs by time since illness onset (hypothesis 4), we compare the interaction effects between marital status and the experience of a chronic condition on the year of onset, with the interaction effects between marital status and the experience of a chronic condition in subsequent years. Again, this is accomplished via Wald tests.

Both models are estimated separately for men and women because the correlates of mental health are known to differ by gender (Pincinelli \& Wilkinson, 2000; van de Velde, Bracke \& Levecque, 2010). We test for gender differences in the estimated effects using analogous models in which all covariates are interacted with gender (see e.g. Gordon, 2015: 324). In these models, the $p$-values on the interactions between gender and the covariates provide the requisite evidence to assess effect heterogeneity by gender.

\section{Empirical evidence}

\section{Dynamic effects of chronic conditions on mental health}

Results from model 1 in table 2 indicate that, when women have a chronic condition on the year of onset, they report worse mental health than when they do not have a condition $(\beta=-4.00$; $p<0.001)$. The adverse effects of having a chronic condition are even stronger when women are observed at a later stage after illness onset $(\beta=-5.44, p<0.001)$. Wald tests confirm that this difference is statistically significant $(p<0.001)$. There is also analogous evidence of differences in mental health with time since illness onset in the male sample. Men's average mental health is 3.2 units $(p<0.001)$ lower when they are in the year of onset of a chronic condition, compared to when they have no chronic conditions. For a chronic condition observed at a subsequent year, the drop in mental health is even more pronounced, of 4.9 units $(p<0.001)$. Again, Wald tests confirm that this difference is statistically significant $(p<0.001)$.

Altogether, these results are consistent with hypotheses 1 (Living with a chronic condition will be associated with poor mental health) and 2a (Chronic illnesses will have a less negative effect on mental health in the year of their onset than in subsequent years). For both men and women, living with a chronic condition is associated with decreases in mental health, and more so at later stages than at the year of onset. Concerning gender differences, Wald tests reveal that the negative impact on 
mental health of having a chronic condition on the year of onset is stronger for women than men $(p<0.001)$. However, there are no gender differences in mental health for the experience of chronic conditions at subsequent stages $(p>0.1)$.

\section{The mental health premium of partnerships revisited}

Results from model 1 are also used to establish whether or not the mental health premium associated with partnerships, particularly, marriages, identified in previous research is also apparent in our Australian sample. The estimated coefficients on the marital status dummy variables in model 1 indicate that women who are divorced, separated or widowed $(\beta=-2.71 ; p<0.001)$, single $(\beta=-1.72 ; p<0.001)$, or in a de facto relationship $(\beta=-0.94 ; p<0.001)$ report poorer mental health than married women. Similarly, men who are divorced, separated or widowed $(\beta=-2.90$; $p<0.001)$, single $(\beta=-1.65 ; p<0.001)$ or in a de facto relationship $(\beta=-0.62 ; p<0.01)$ report poorer mental health than married men. Results from Wald tests further reveal that men and women in de facto relationships report better mental health than their counterparts who are single or divorced, separated or widowed $(p<0.01$ to $p<0.001$ across all pairwise comparisons).

Therefore, model 1 provides evidence that is consistent with previous literature: for both men and women there is a mental health premium associated with being partnered, which is stronger for married individuals. Results of Wald tests comparing the predicted effects on the marital status dummy variables between men and women yielded no evidence of gender differences.

\section{Marital status as a dynamic moderator of the chronic condition/mental health association}

Model 2 is used to test our remaining hypotheses. The results for women provide evidence of moderation in the effects of our measure of chronic conditions on mental health by marital status and that, to some extent, the pattern of moderation varies with time since illness onset. Five of the six interaction terms are statistically significant, confirming that being married (the reference category) is an important moderator of the chronic illness/mental health association. However, Wald tests provide little evidence of moderation for de facto relationships, as few of these tests are statistically significant. To get an overall picture of the results, it is helpful to interpret the main and interaction effects in conjunction. When doing so, the mental health impacts of being in the onset year of a chronic condition (compared to not having a condition) are largest when women are single ( -5.6 units), followed by when they are divorced, separated or widowed (-3.9 units), in a de facto relationship ( -5 units), and married (-3.1 units). This ordering changes slightly when one considers the mental health impacts of being in a subsequent year of a chronic condition (compared to not having a condition). For this category, the largest mental health 'penalties' occur when women are single (-6.7 units), followed by when they are in a de facto relationship (-6 units), divorced, separated or widowed ( -5.4 units), and married ( -4.9 units).

There is also evidence of moderation by marital status amongst men. As for women, the pattern of results provides strong evidence of moderation for marriage, and only weak evidence for de facto relationships. Taking the main and interaction effects together, being in the onset year of a chronic condition takes the greatest toll on men's mental health when they are single ( -4.5 units), followed by when they are divorced, separated or widowed (-3.9 units), in a de facto relationship (-3.8 units), and married (-2.4 units). Chronic conditions observed after the year of onset have the largest negative effects on men's mental health when they are single (-6.9 units), followed by when they are in a de facto relationship (-6.2 units), married ( -4.4 units), and divorced, separated or widowed ( -4.1 units). The results of model 2 are visually represented in figure 1 as marginal effects, with covariate values set at their means and random effects at zero.

The protection on mental health of being married (compared to being single) was stronger when individuals experienced chronic conditions than when they did not. While this marriage protection effect was apparent amongst women (-3.8 units) and men (-2.1 units) experiencing no such conditions, its magnitude was larger for women ( -6.2 units) and men ( -4.1 units) in the year of onset of a condition, and for women ( -5.6 units) and men (-4.6 units) experiencing a condition beyond the year of onset.

Altogether, these results provide mixed evidence for our third hypothesis (The negative mental health impacts of chronic conditions will be stronger 
Table 2. Random-effect models of mental health

\begin{tabular}{|c|c|c|c|c|c|c|}
\hline & \multicolumn{3}{|c|}{ Model 1} & \multicolumn{3}{|c|}{ Model 2} \\
\hline & Women & Men & Gender diff. & Women & Men & Gender diff. \\
\hline \multicolumn{7}{|l|}{ Has a chronic condition (ref. No) } \\
\hline On the year of onset & $-4.00^{* * *}$ & $-3.21^{* * *}$ & $* * *$ & $-3.14^{* * *}$ & $-2.44^{* * *}$ & * \\
\hline On a subsequent year & $-5.44^{* * *}$ & $-4.94^{* * *}$ & n.s. & $-4.90^{* * *}$ & $-4.36^{* * *}$ & n.s. \\
\hline \multicolumn{7}{|l|}{ Marital status (ref. Married) } \\
\hline In a de facto relationship & $-0.94^{* * *}$ & $-0.62^{* *}$ & n.s. & $-0.65^{* *}$ & -0.31 & n.s. \\
\hline Divorced, separated or widowed & $-2.71^{* * *}$ & $-2.90^{* * *}$ & n.s. & $-2.57^{* * *}$ & $-2.90^{* * *}$ & n.s. \\
\hline Single (never married) & $-1.72^{* * *}$ & $-1.65^{* * *}$ & n.s. & $-1.34^{* * *}$ & $-1.23^{* * *}$ & n.s. \\
\hline \multicolumn{7}{|l|}{ Marital status \& chronic condition interactions } \\
\hline Condition, onset $*$ In a de facto relationship & & & & $-1.84^{* * *}$ & $-1.37^{* *}$ & n.s. \\
\hline Condition, onset * Divorced, separated or widowed & & & & $-0.77^{(*)}$ & $-1.45^{* *}$ & n.s. \\
\hline Condition, onset $*$ Single (never married) & & & & $-2.48^{* * *}$ & $-2.07^{* * *}$ & n.s. \\
\hline Condition, no onset $*$ In a de facto relationship & & & & $-1.12^{*}$ & $-1.80^{* * *}$ & n.s. \\
\hline Condition, no onset * Divorced, separated or widowed & & & & -0.49 & 0.26 & n.s. \\
\hline Condition, no onset $*$ Single (never married) & & & & $-1.83^{* * *}$ & $-2.51^{* * *}$ & n.s. \\
\hline \multicolumn{7}{|l|}{ Control variables } \\
\hline Age (in years) & $0.14^{* * *}$ & $0.08^{* * *}$ & *** & $0.14^{* * *}$ & $0.08^{* * *}$ & *** \\
\hline \multicolumn{7}{|l|}{ Number of children ever had (ref. Zero) } \\
\hline One & 0.14 & $-1.02^{* * *}$ & *** & 0.19 & $-0.96^{* * *}$ & *** \\
\hline Two or more & $-1.05^{* * *}$ & $-1.44^{* * *}$ & n.s. & $-1.01^{* * *}$ & $-1.39^{* * *}$ & n.s. \\
\hline \multicolumn{7}{|l|}{ Educational attainment (ref. Degree) } \\
\hline Below Year 12 & $-1.32^{* * *}$ & $0.87^{* *}$ & *** & $-1.36^{* * *}$ & $0.84^{* *}$ & $* * *$ \\
\hline Year 12 & $-1.06^{* * *}$ & 0.29 & *** & $-1.10^{* * *}$ & 0.26 & *** \\
\hline Professional qualification & $-1.05^{* * *}$ & 0.09 & $* *$ & $-1.08^{* * *}$ & 0.08 & ** \\
\hline \multicolumn{7}{|l|}{ Employment status (ref. Employed) } \\
\hline Unemployed & $-1.51^{* * *}$ & $-2.70^{* * *}$ & $* *$ & $-1.49^{* * *}$ & $-2.68^{* * *}$ & $* *$ \\
\hline Not in the labour force & $-1.03^{* * *}$ & $-1.48^{* * *}$ & $*$ & $-1.03^{* * *}$ & $-1.49^{* * *}$ & $*$ \\
\hline Household income (in $A \$ 10,000$ s) & $0.05^{* * *}$ & $0.08^{* * *}$ & $* *$ & $0.05^{* * *}$ & $0.08^{* * *}$ & $* *$ \\
\hline
\end{tabular}


Table 2. Random-effect models of mental health (cont.)

Ethno-migrant background (ref. Australian, not Ind.)

Australian, Indigenous

Migrant, main English-speaking country

$-4.47^{* * *}-1.90^{*}$

$0.24 \quad 0.59$

0.59
$-2.76^{* * *}$

n.s.

$-4.39^{* *}$

$-1.84^{*}$

Migrant, other country

$-2.70^{* *}$

2.76

n.s.

0.22

0.59

Wald tests

$B$ (cond_onset) $=B$ (cond_later)

$B($ de facto $)=B($ divorced $)$

$B($ de facto $)=B($ single $)$

$B($ divorced $)=B($ single $)$

$B$ (cond_onset*de facto $)=B$ (cond_onset*divorced)

$B$ (cond_onset*defacto $)=B$ (cond_onset*single)

$B$ (cond_onset*divorced) $=B$ (cond_onset*single)

$B$ (cond_later*de facto $)=B$ (cond_later*divorced)

$B$ (cond_later*de facto $)=B$ (cond_later*single)

$B$ (cond_later*divorced $)=B$ (cond_later*single)

\begin{tabular}{|c|c|c|c|c|}
\hline $\mathrm{N}$ (observations) & 96,138 & 84,426 & 96,138 & 84,426 \\
\hline N (individuals) & 13,859 & 12,877 & 13,859 & 12,877 \\
\hline $\mathrm{R}^{2}$ & 0.097 & 0.083 & 0.097 & 0.084 \\
\hline
\end{tabular}

Notes: HILDA Survey data, 2002-2015. Outcome variable: MHI-5 (transformed to range from 0 to 100). Standard errors are clustered for the nesting of observations within households. Gender differences are tested via models in which all variables are interacted with gender. Significance levels: $(*)$ p<0.1, * $p<0.05, * * p<0.01, * * * p<0.001$, n.s. $p>0.1$. 
Figure 1. Predicted mental health by gender, marital status and time since illness onset

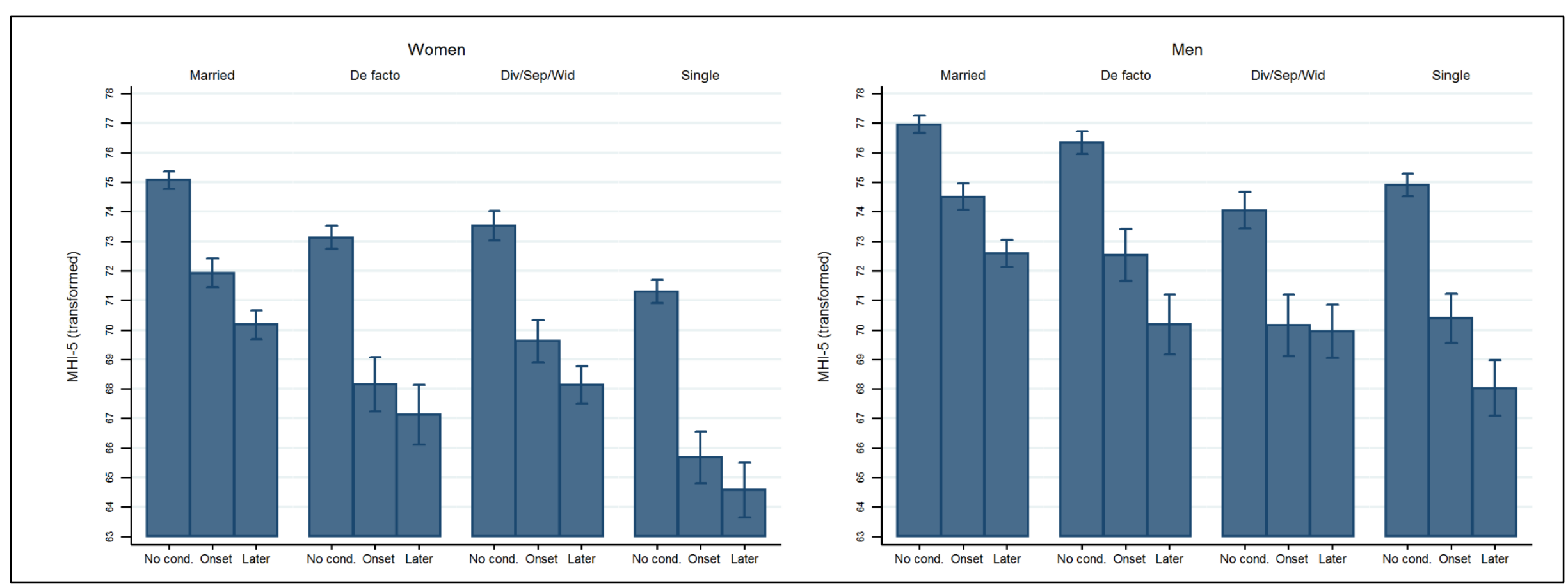

Notes: HILDA Survey data, 2002-2015. Whiskers denote 95\% confidence intervals. Based on results from models 3 (women) and 4 (men) in table 2. Values of the covariates are set at their means and random effects are set at zero. 
amongst unpartnered than partnered individuals - particularly married individuals) and our fourth hypothesis (The protective effect of being partnered on the mental health of individuals living with a chronic condition will decline with time since illness onset). For both men and women, being married (and to a lesser extent being in a de facto relationship) buffers the negative mental health effects of living with a chronic condition. This is consistent with hypothesis 3 . However, against the predictions in hypothesis 4 , we find little evidence that the protective effect of partnerships on mental health changes as time since illness onset progresses.

\section{Discussion and conclusion}

In this paper, we have drawn on the stress process model to examine the longitudinal associations between marital status, chronic conditions and mental health. We innovated by testing the role of partnership as a factor moderating the negative association between chronic conditions and mental health in a longitudinal context and considering gender differences. Our empirical analyses relied on 15 years of panel data from a national sample, the Household, Income and Labour Dynamics in Australia Survey, and random-effect panel regression models.

We began our empirical analysis by testing a confirmatory hypothesis on the direct effects of chronic conditions on mental health. This posed that living with a chronic condition should be associated with poor mental health (hypothesis 1). Consistent with previous studies and in favour of this hypothesis, we found that in our sample chronic conditions were indeed related to poorer mental health for both men and women (Hollingshaus \& Utz, 2013; Polsky et al., 2005; Pudrovska, 2010). Hypotheses 2a and 2b delved into the longitudinal dimensions of this association: hypothesis $2 a$ suggested a 'cumulative disadvantage' scenario (chronic illnesses having less negative effects on mental health in the year of onset than in subsequent years), whereas hypothesis $2 b$ suggested $a$ 'biographical disruption' scenario (chronic illnesses having more negative effects on mental health in the year of onset than in subsequent years). While previous studies have provided mixed findings regarding the dynamics of chronic illness and mental health (De Ridder et al., 2008; van't Spijker, Trijsburg \& Duivenvoorden, 1997), our findings are clearly consistent with a 'cumulative disadvantage' situation: for both men and women, respondents reported worse mental health levels beyond the year of the illness onset, ceteris paribus. Concerning effect magnitude, for chronic conditions experienced in the year of onset, the estimates amount to $23 \%$ (women) and $19 \%$ (men) of a standard deviation in self-reported mental health. The analogous figures for chronic conditions experienced in subsequent years are $31 \%$ (women) and $30 \%$ (men) of a standard deviation.

Our results were also telling in regards to how being partnered, and particularly being married, is associated with a mental health premium in Australia. We found evidence that, all else being equal, men and women in partnerships reported better mental health levels relative to men and women who were single, divorced, separated and widowed (Rendall et al., 2011). There were also statistically significant differences in mental health levels between marital and de facto unions, with men and women in de facto relationships reporting poorer mental health than their married counterparts. These differences in mental health by marital status were generally substantially significant. For example, the coefficient on being divorced, separated or widowed amounts to about $15 \%$ of the measure's standard deviation for women and $17 \%$ for men.

Next, we innovated by explicitly considering whether and how marital status acts as a moderator of the chronic conditions/mental health nexus (hypothesis 3), and whether the stress-buffering role of partners persists with time since illness onset (hypothesis 4). As predicted by the stress process model and consistent with hypothesis 3, we found that for both men and women the negative mental health impacts of chronic conditions were stronger amongst unpartnered than partnered individuals. As such, partners do seem to act as protective resources or 'stress buffers' in the 
face of chronic conditions. While this applied to both marital and de facto unions, the estimated protective effects were more pronounced amongst the former than the latter. Importantly, the marriage protection effect was stronger amongst women and men experiencing a chronic condition, irrespective of its stage, than amongst women and men with no conditions.

When considering temporal dynamics in the panel data, we found less support for hypothesis 4: for both men and women, the protective effect of partnerships against the negative mental health effects of chronic conditions remained stable with time since illness onset. This suggests that mental health advantages amongst the chronically ill conferred by their partners are durable. The magnitude of the interaction effects between marital status and chronic illnesses was not negligible. For example, the protective effect of being married relative to single in the year of illness onset amounts to between 14\% (women) and $12 \%$ (men) of a standard deviation in the mental health measure. The analogous figures for subsequent years after illness onset were $10 \%$ (women) and $15 \%$ (men). The size of these interaction effects is thus comparable to or stronger than those of factors to which the literature has paid more attention. For example, it is similar to the estimated effects of employment status and greater than those of educational qualifications and the number of children. Altogether, these findings highlight the importance of considering partners and the support they confer as a continuing resource that has the potential to ease the deleterious effects of having a chronic condition on mental health. Such protective effects have significant ramifications, as mental health is a crucial factor promoting adherence to medical recommendations amongst individuals with a chronic condition and has implications for disease management, recovery and mortality (DiMatteo, Lepper, \& Croghan, 2000; Carney, Freeland, Miller, \& Jaffe, 2002).

Given known differences in the emotional and instrumental resources that men and women bring into relationships, we tested for gender differences. We found some evidence of gender differences in the negative impact on mental health of having a chronic condition on the year of onset, which was stronger for women than men. However, this difference faded at subsequent years. There was however only weak evidence of differences by gender in the direct effect of being in a partnership on mental health, or in the protective effects of partnerships on the mental health of the chronically ill. Concerning the latter, the protective effects of marriage relative to singlehood and de facto relationships seemed to become stronger in the years after illness onset amongst men, but weaker amongst women. This pattern of results is consistent with reports of gender differences in spousal caregiving, with women being more likely than men to provide care and to provide greater amounts of care (Moen, Robison, \& Fields, 1994; Goldzweig et al., 2009; Katz et al., 2000; Noël-Miller, 2010; Spitze \& Ward, 2000). However, in our data, these differences were neither statistically nor substantially significant. We therefore conclude that the relationships of interest were generally consistent for men and women in our Australian sample.

Despite our several contributions to the literature, there are limitations to our study that point towards avenues for future refinement. First, as with others before us (see e.g. Hollinghaus \& Utz, 2013; Patten, 1999; Schnittker, 2005), we could only use a coarse, binary measure of whether or not individuals have a chronic condition. This did not distinguish the number of conditions, their nature, or the severity of the symptoms. Hence, our results are a broad starting point, and may mask heterogeneity in how marital status and mental health relate to each other across different types and experiences of chronic conditions. Second, while we are amongst the first to study the relationships of interest longitudinally, data shortcomings forced us to simplify time since illness onset into two components: (i) the year of onset and (ii) subsequent years. This is chiefly due to a lack of information on the specific year in which a chronic condition had its onset for individuals who entered the panel with a pre-existing chronic condition. As a result, we could not 
discern more finely grained trends in mental health without losing a large portion of our analytic sample. Third, while our results are informative of the main and interactive effects of chronic conditions and marital status on mental health, they are silent about the mechanisms driving these associations. We interpret our findings as suggesting that partnerships (and especially marriages) are an important source of emotional, instrumental and/or financial support for the chronically ill. Subsequent studies may delve further into the types of support that partners provide, and which of these are more crucial to the mental health of their significant others. Qualitative studies may be particularly well-suited for this task. Fourth, the results reported here are associations, and may not represent causal relationships. In particular, it is possible that some of the protective effects of partnerships/marriages are due to selection into these states. For example, individuals who enjoy better physical and mental health may be more likely to find and retain a partner. However, recent research finds that the positive health effects of having a partner often remain after accounting for such selection (Blekesuane, 2008; Guner, Kulikova, \& Llull, 2014), suggesting that selection is unlikely to single-handedly explain our results.

Despite these limitations, our findings have significant implications for health policy and practice. The pattern of results found for partnerships (particularly marriages) indicates that being diagnosed and living with a chronic illness has more detrimental effects on the mental health of people who are unpartnered. The protective effect of partnerships was also found to be durable. These are policy-relevant findings, as they point towards the need for institutional intervention to ensure that the mental health levels of unpartnered individuals remain stable over the duration of their illness. Provision of external support to these individuals to compensate for the absence of a partner should be considered. Interventions might include redefining laws and policies that disadvantage unpartnered individuals (e.g. insurance policies), implementation of support groups and information sessions where individuals learn about how to manage their illness, or direct provision of care or economic transfers to cover its cost by government (Helgeson \& Cohen, 1996; Meyer \& Mark, 1995).

While the magnitude of the individual effects on the protective effects of partnerships in our findings is moderate, the population accumulation of such effects constitutes a public health problem. The associated economic and social costs at the national level are also likely to grow in the future: the population in advanced economies such as Australia is ageing, chronic illness is prevalent, and the incidence of partnerships (particularly marriages) is in decline (Australian Bureau of Statistics, 2015; Jain, 2007; Kennedy \& Ruggles, 2014). Collectively, these patterns suggest that, while population ageing may lead to more people living with chronic illness and for longer spells of time, the benefits conferred by having a partner in buffering the mental strain of a chronic condition will apply to a shrinking portion of the population. Addressing these issues is thus an important challenge for social and public health policy, and will require more scholarly attention. 


\section{Acknowledgements}

This paper uses unit record data from the Household, Income, and Labour Dynamics in Australia (HILDA) Survey. The HILDA Project was initiated and is funded by the Australian Government Department of Social Services (DSS) and is managed by the Melbourne Institute of Applied Economic and Social Research (Melbourne Institute). The findings and views reported in this paper, however, are those of the authors and should not be attributed to either DSS or the Melbourne Institute. This research was supported by the Australian Research Council Centre of Excellence for Children and Families over the Life Course (project number CE140100027). The Centre is administered by the Institute for Social Science Research at The University of Queensland, with nodes at The University of Western Australia, The University of Melbourne and The University of Sydney.

\section{References}

Adelman, R. D., Lyubov L., Tmanova, D. D., Dion, S., \& Lachs, M. (2014). Caregiver Burden: A Clinical Review. JAMA, 311(10), 1052-1060. https://doi.org/10.1001/jama.2014.304

Allen, S. M., Goldscheider, F., \& Ciambrone, D. A. (1999). Gender roles, marital intimacy, and nomination of spouse as primary caregiver. The Gerontologist, 39(2), 150-158. https://doi.org/10.1093/geront/39.2.150

August, K. J. \& Sorkin, D. H. (2010). Marital Status and Gender Differences in Managing a Chronic Illness: The Function of Health-Related Social Control. Social Science and Medicine, 71(10), 1831-1838. https://doi.org/10.1016/j.socscimed.2010.08.022

Australian Bureau of Statistics (ABS). (2015). Marriages and Divorces, Australia, 2014 (cat. no. 331.0). Canberra: ABS.

Australian Institute of Health and Welfare (AIHW). (2014). Chapter 4. Chronic Disease-Australia's Biggest Health Challenge (Australia's health series no.14, Cat.No.AUS178). Canberra: AIHW.

Avison, W. R. \& Turner, R. J. (1988). Stressful Life Events and Depressive Symptoms: Disaggregating the Effects of Acute Stressors and Chronic Strains. Journal of Health and Social Behavior, 29(3), 253-264. https://doi.org/10.2307/2137036

Berkman, L. F. \& Glass, T. (2000). Social Integration, Social Networks, Social Support, and Health. Social Epidemiology, 1, 137-173.

Bierman, A. (2012). Functional Limitations and Psychological Distress: Marital Status as Moderator. Society and Mental Health, 2(1), 35-52. https://doi.org/10.1177/2156869312442884

Blekesuane, M. (2008). Partnership Transitions and Mental Distress: Investigating Temporal Order. Journal of Marriage and Family, 70(4), 879-890. https://doi.org/10.1111/j.17413737.2008.00533.x

Booth, A. \& Johnson, D. R. (1994). Declining Health and Marital Quality. Journal of Marriage and Family, 56(1), 218-223. https://doi.org/10.2307/352716

Bury, M. (1982). Chronic Illness as Biographical Disruption. Sociology of Health and IIIness, 4(2), 16782. https://doi.org/10.1111/1467-9566.ep11339939

Carney, R. M., Freedland, K., Miller, G., \& Jaffe, A. (2002). Depression as a Risk Factor for Cardiac Mortality and Morbidity: A Review of Potential Mechanisms. Journal of Psychosomatic Research, 53, 897-902. https://doi.org/10.1016/S0022-3999(02)00311-2

Cho, C.-Y., Alessi, C., Cho, M., Aronow, H., Stuck, A., Rubenstein, L., \& Beck, J. (1998). The Association between Chronic Illness and Functional Change among Participants in a Comprehensive Geriatric Assessment Program. Journal of the American Geriatrics Society, 46(6), 677-682. https://doi.org/10.1111/i.1532-5415.1998.tb03800.x

Clarke, D. M. \& Currie, K. C. (2009). Depression, Anxiety and their Relationship with Chronic Diseases: A Review of the Epidemiology, Risk and Treatment Evidence. The Medical Journal of Australia, 190(7), 54-60. 
Cohen, S. (2004). Social Relationships and Health. American Psychologist, 59(8), 676-684. https://doi.org/10.1037/0003-066X.59.8.676

Cohen, S. \& Wills, T. A. (1985). Stress, Social Support, and the Buffering Hypothesis. Psychological Bulletin, 98(2), 310-357.

Coombs, R. H. (1991). Marital Status and Personal Well-Being: A Literature Review. Family Relations, 40(1), 97-102. https://doi.org/10.2307/585665

De Ridder, D., Geenen, R., Kuijer, R., \& van Middendorp, H. (2008). Psychological Adjustment to Chronic Disease. The Lancet, 372(9634), 246-55. https://doi.org/10.1016/S0140$\underline{6736(08) 61078-8}$

DiMatteo, M. R., Lepper, H., \& Croghan, T. W. (2000). Depression is a Risk Factor for Noncompliance with Medical Treatment: Meta-Analysis of The Effects of Anxiety and Depression on Patient Adherence. Archives of Internal Medicine, 160(14), 2101-7. https://doi.org/10.1001/archinte.160.14.2101

Dunlop, D. D., Lyons, J. S., Manheim, L., Song, J. \& Chang, R. W. (2004). Arthritis and Heart Disease as Risk Factors for Major Depression: The Role of Functional Limitation. Medical Care, 42(6), 502-511. https://doi.org/10.1097/01.mlr.0000127997.51128.81

England, P. (2010). The Gender Revolution: Uneven and Stalled. Gender \& Society, 24(2), 149-166. https://doi.org/10.1177/0891243210361475

Essue, B., Kelly, P., Roberts, M., Leeder, S., \& Jan, S. (2011). We Can't Afford My Chronic Illness! The Out-Of-Pocket Burden Associated with Managing Chronic Obstructive Pulmonary Disease in Western Sydney, Australia. Journal of Health Services Research \& Policy, 16(4), 226-231. https://doi.org/10.1258/ihsrp.2011.010159

Frech, A., \& Williams, K. (2007). Depression and the Psychological Benefits of Entering Marriage. Journal of Health and Social Behavior, 48(2), 149-163. https://doi.org/10.1177/002214650704800204

Goldzweig, G., Hubert, A., Walach, N., Bremmer, B., Perry, S., Andritsch, E., \& Baider, L. (2009). Gender and Psychological Distress among Middle- and Older-Aged Colorectal Cancer Patients and their Spouses: An Unexpected Outcome. Critical Reviews in Oncology/Hematology, 70(1), 71-82. https://doi.org/10.1016/i.critrevonc.2008.07.014

Gordon, R. (2015). Regression Analysis for the Social Sciences ( $2^{\text {nd }}$ Edition). New York: Routledge.

Guner, N., Kulikova, Y., \& Llull, J. (2014). Does Marriage Make You Healthier? (IZA Discussion Paper No. 8633). Bonn: IZA.

Helgeson, V. S. \& Cohen, S. (1996). Social Support and Adjustment to Cancer: Reconciling Descriptive Correlational, and Intervention Research. Healthy Psychology, 15(2), 135-48. https://doi.org/10.1037/0278-6133.15.2.135

Holahan, C. K., Holahan, C., \& Belk, S. S. (1984). Adjustment in Aging: The Roles of Life Stress, Hassles and Self-Efficacy. Health Psychology, 3(4), 315-328. https://doi.org/10.1037/0278$\underline{6133.3 .4 .315}$

Hollingshaus, M. S. \& Utz, R. (2013). Depressive Symptoms Following the Diagnosis of Major Chronic Illness. Society and Mental Health, 3(1), 22-39. https://doi.org/10.1177/2156869312464788

House, J, S., Landis, K., \& Umberson, D. (1988). Social Relationships and Health. Science, 241(4865), 540-5. https://doi.org/10.1126/science.3399889

Jain, S. (2007). Lifetime Marriage and Divorce Trends. (Australian Social Trends, 2007). Canberra: Australian Bureau of Statistics.

Kasl, S. V. \& Jones, B. A. (2000). The Impact of Job Loss and Retirement on Health. In: L. F. Berkman \& I. Kawachi (eds.). Social Epidemiology. New York: Oxford University Press, pp. 118-136.

Katz, S. J., Kabeto, M., \& Langa, K. M. (2000). Gender Disparities in the Receipt of Home Care for Elderly People with Disability in the United States. JAMA, 284, 3022-3027. https://doi.org/10.1001/jama.284.23.3022 
Kennedy, S. \& Ruggles, S. (2014). Breaking up is hard to count: The rise of divorce in the United States, 1980-2010. Demography, 51(2), 587-598. https://doi.org/10.1007/s13524-0130270-9

Kristofferzon, M.-L., Lofmark, R., \& Carlsson, M. (2003). Myocardial Infarction: Gender Differences in Coping and Social Support. Journal of Advanced Nursing, 44(4), 360-374. https://doi.org/10.1046/i.0309-2402.2003.02815.x

Liu, H. \& Umberson, D. (2008). The times they are changin': Marital status and health differentials from 1972 to 2003. Journal of Health and Social Behavior, 49, 239-253. https://doi.org/10.1177/002214650804900301

Mechanic, D. \& Volkart, E. H. (1961). Stress, Illness Behavior, and the Sick Role. American Sociological Review, 26(1), 51-58. https://doi.org/10.2307/2090512

Meyer, T. J. \& Mark, M. M. (1995). Effects of psychosocial interventions with adult cancer patients: A meta-analysis of randomized experiments. Health Psychology, 14(2), 101-108. https://doi.org/10.1037/0278-6133.14.2.101

Moen, P., Robison, J., \& Fields, V. (1994). Women's Work and Caregiving Roles: A Life Course Approach. The Journals of Gerontology. Series B, Psychological Sciences and Social Sciences, 49(4), S176-S186. https://doi.org/10.1093/geronj/49.4.S176

Murray, J. E. (2000). Marital protection and marital selection: Evidence from a historical-prospective sample of American men. Demography, 37, 511-521. https://doi.org/10.1353/dem.2000.0010

Newman, S., Steed, L., \& Mulligan, K. (2004). Self-Management Interventions for Chronic Illness. The Lancet, 364(9444), 1523-37. https://doi.org/10.1016/S0140-6736(04)17277-2

Noël-Miller, C. (2010). Longitudinal Changes in Disabled Husbands' and Wives' Receipt of Care. The Gerontologist, 50(5), 681-693. https://doi.org/10.1093/geront/gnq028

Noël-Miller, C. (2011). Partner Caregiving in Older Cohabiting Couples. The Journals of Gerontology. Series B, Psychological Sciences and Social Sciences, 66B(3), 341-53. https://doi.org/10.1093/geronb/gbr027

Patten, S. B. (1999). Long-Term Medical Conditions and Major Depression in the Canadian Population. Canadian Journal of Psychiatry, 44(2), 151-157. https://doi.org/10.1177/070674379904400205

Pearlin, L. I. (1989). The Sociological Study of Stress. Journal of Health and Social Behavior, 30(3), 241-256. https://doi.org/10.2307/2136956

Pearlin, L. I., Menaghan, E. G., Lieberman, M. A., \& Mullan, J. T. (1981). The Stress Process. Journal of Health and Social Behavior, 22(4), 337-356. https://doi.org/10.2307/2136676

Perry, B. L. (2011). The Labeling Paradox: Stigma, the Sick Role, and the Social Networks in Mental Illness. Journal of Health and Social Behavior, 52(4), 460-477. https://doi.org/10.1177/0022146511408913

Pincinelli, M., \& Wilkinson, G. (2000). Gender differences in depression: Critical review. British Journal of Psychiatry, 177(6), 486-492. https://doi.org/10.1192/bjp.177.6.486

Pitceathly, C. \& Maguire, P. (2003). The Psychological Impact of Cancer on Patients' Partners and Other Key Relatives: A Review. European Journal of Cancer, 39(11), 1517-1524. https://doi.org/10.1016/S0959-8049(03)00309-5

Polsky, D., Doshi, J., Marcus, S., Oslin, D., Rothbard, A., Thomas, N., \& Thompson, C. L. (2005). LongTerm Risk for Depressive Symptoms after a Medical Diagnosis. Archives of Internal Medicine, 165(11), 1260-66. https://doi.org/10.1001/archinte.165.11.1260

Pudrovska, T. (2010). Why Is Cancer More Depressing for Men than Women among Older White Adults? Social Forces, 89(2), 535-558. https://doi.org/10.1353/sof.2010.0102

Rendall, M., Weden, M. M., Favreault, M. M., \& Waldron, H. (2011). The Protective Effect of Marriage for Survival: A Review and Update. Demography, 48(2), 481-506. https://doi.org/10.1007/s13524-011-0032-5 
Schafer, M. H. \& Koltai, J. (2015). Cancer Diagnosis and Mental Health among Older White Adults: Moderating Role for Social Networks. Society and Mental Health, 5(3), 282-202. https://doi.org/10.1177/2156869315577631

Schnittker, J. (2005). Chronic Illness and Depressive Symptoms in Late Life. Social Science \& Medicine, 60, 13-23. https://doi.org/10.1016/i.socscimed.2004.04.020

Serido, J., Almeida, D. M., \& Wethington, E. (2004). Chronic Stressors and Daily Hassles: Unique and Interactive Relationships with Psychological Distress. Journal of Health and Social Behavior, 45(1), 17-33. https://doi.org/10.1177/002214650404500102

Spitze, G. \& Ward, R. R. (2000). Gender, Marriage, and Expectations for Personal Care. Research on Aging, 22(5), 451-469. https://doi.org/10.1177/0164027500225001

Stanton, A. L., Revenson, T. A., \& Tennen, H. (2007). Health Psychology: Psychological Adjustment to Chronic Disease. Annual Review of Psychology, 58, 565-92. https://doi.org/10.1146/annurev.psych.58.110405.085615

Summerfield, M., Freidin, S., Hahn, M., La, N, Li, N., Macalalad, N., O'Shea, M., Watson, N., Wilkins, R. \& Wooden, M. (2016). HILDA User Manual-Release 15. Melbourne Institute of Applied Economic and Social Research, University of Melbourne.

Thomeer, M. B., Reczek, C., \& Umberson, D. (2015). Gendered Emotion Work around Physical Health Problems in Mid- and Later-life Marriages. Journal of Aging Studies; 32, 12-22. https://doi.org/10.1016/j.jaging.2014.12.001

Umberson, D. (1987). Family Status and Health Behaviors: Social Control as a Dimension of Social Integration. Journal of Health and Social Behavior, 28(3), 306-319. https://doi.org/10.2307/2136848

Umberson, D. (1992). Gender, marital status, and the social control of health behaviour. Journal of Health and Social Behavior, 34, 907-917.

van de Velde, S., Bracke, P., \& Levecque, K. (2010). Gender differences in depression in 23 European countries. Cross-national variation in the gender gap in depression. Social Science \& Medicine, 71(2), 305-313. https://doi.org/10.1016/i.socscimed.2010.03.035

van't Spijker, A., Trijsburg, R. W., \& Duivenvoorden, H. J. (1997). Psychological Sequelae of Cancer Diagnosis: A Meta-Analytical Review of 58 Studies after 1980. Psychosomatic Medicine, 59(3), 280-293. https://doi.org/10.1097/00006842-199705000-00011

Ware, J. E., \& Sherbourne, C. D. (1992). The MOS 36-Item Short-Form Health Survey (SF-36):I. Conceptual Framework and Item Selection. Medical Care, 30(6), 473-483. https://doi.org/10.1097/00005650-199206000-00002

Wiik, K. A., Bernhardt, E., \& Noack, T. (2009). A Study of Commitment and Relationship Quality in Sweden and Norway. Journal of Marriage and Family, 71(3), 465-477. https://doi.org/10.1111/j.1741-3737.2009.00613.x

Wiik, K. A., Keizer, R., \& Lappegård, T. (2012). Relationship Quality in Marital and Cohabiting Unions across Europe. Journal of Marriage and Family, 74(3), 389-398. https://doi.org/10.1111/j.17413737.2012.00967.x

Williams, K. (2003). Has the future of marriage arrived? A contemporary examination of gender, marriage, and psychological well-being. Journal of Health and Social Behavior, 44(4), 470487. https://doi.org/10.2307/1519794

Willig, C. (2011). Cancer Diagnosis as Discursive Capture: Phenomenological Repercussions of Being Positioned within Dominant Constructions of Cancer. Social Science \& Medicine, 73, 897903. https://doi.org/10.1016/j.socscimed.2011.02.028

Watson, N., \& Wooden, M. (2012). The HILDA Survey: A Case Study in the Design and Development of a Successful Household Panel Survey. Longitudinal and Life Course Studies, 3(3), 369-381 


\section{Endnotes}

1. For example, $86 \%$ of people aged 65 years and older in the US and $78 \%$ of people in the same age group in Australia have at least one chronic condition (AlHW 2014; Ward, Schiller, and Goodman 2014).

2. We opt for random-effect instead of fixed-effect models because low rates of within individual change in marital status and the experience of chronic conditions make the latter inefficient. Sensitivity analyses conducted using fixed-effect models yield a very similar pattern of results, and are available from the authors upon request.

3. The standard errors in all regression models are adjusted for the nesting of individuals within households. 